\section{Closed Femoral Shaft Fracture Associated With Superficial Femoral Artery Thrombosis in a Child}

\section{Abstract}

Femur fractures are common in pediatric population. However, traumatic vascular injuries secondary to femoral shaft fractures were rarely reported in the literature. Major signs of the vascular injury are including pulselessness, enlarging pulsatile haematoma, bruit, thrill, haemorrhage and acute ischemia. Vascular injury may present even if in the presence of the distal pulses of the limb. We report a case of 5 year old child who presented with superficial femoral artery thrombosis due to proximal femoral shaft fracture. We emphasize the significance of suspicion of vascular injuries associated with femoral shaft fractures in pediatric population and the influence of the current research on the future studies.

Keywords: Femoral shaft fracture; Vascular injury; Surgical treatment

\author{
Serkan Burc Deser and \\ Mustafa Kemal Demirag \\ 19 Mayis University, Faculty of Medicine, \\ Department of Cardiovascular Surgery, \\ Samsun, Turkey
}

Corresponding author: Serkan Burc Deser

\section{sbd983@yahoo.com}

Department of Cardiovascular Surgery, 19 Mayis University, Faculty of Medicine, 55139 Samsun, Turkey.

Tel: 00905385012010

Fax: 00903624576091

Citation: Deser SB, Demirag MK. Closed Femoral Shaft Fracture Associated With Superficial Femoral Artery Thrombosis in a Child. J Vasc Endovasc Surg. 2016, 1:3.

\section{Introduction}

Femur fractures are common in paediatric population and femoral shaft fractures have an incidense of $1.4-1.7 \%$ of all paediatric fractures. These are often seen in boys rather than girls. Most of the fractures are transverse, non-comminuted and closed [1]. The mechanism of the femoral shaft fracture for older children is associated with high-energy transmission such as traffic accidents. Combined femoral shaft fracture and arterial injuries of the limbs are infrequent, however, vascular injuries associated with femoral shaft fractures and dislocations have a high morbidity. Common femoral or superficial femoral artery injuries lead to acute limb ischaemic due to restriction of the blood flow by intramural hematoma, spasm, stretch injury, intimal disruption, arteriosclerotic plaque disruption and haemorrhage.

In summary, amputation is an undesired outcome. Amputation rate ranges from $5 \%$ to $40 \%$ even though, successful restoration of the arterial circulation [2].

We report a case of 5 year old child who presented with superficial femoral artery (SFA) thrombosis due to proximal femoral shaft fracture and we emphasize the significance of suspicion of vascular injuries associated femoral shaft fractures.

\section{Case Report}

A 5-year-old man was presented with a right proximal femoral fracture in a traffic accident and he was admitted to Ondokuz
Mayis University Hospital, Samsun, Turkey. On admission, his temperature was 36.5 centigrade celsius, blood pressure was $120 / 80 \mathrm{mmHg}$, pulse was 110 beats/min, respiratory rate was 12 , oxygen saturation while breathing room air was $99 \%$. His physical examination revealed a mass at his right thigh, diminished pulses, capilary refill disruption, coldness and paleness on his right lower limb. No other significant injuries were noted. Direct graphy of the right thigh revealed fracture on the right femoral shaft (Figure 1). Doppler ulrasound (US) revealed thrombus on the superficial femoral artery on the injury site. Routine preparation for surgery was made and informed consent was taken. Close reduction of the femur was done, however, distal pulses were absent and were confirmed with the manuel doppler US. We performed right femoral embolectomy with a $4 \mathrm{~F}$ fogarty thrombectomy catheter. Fresh thrombus material was gained and distal flow was provided which was confirmed with the presence of the distal pulses and the manuel doppler US. The post operative course was uneventful and he was discharged with oral antocoagulation therapy. Six months later he was doing well with stable vital signs and no ischemia on follow up.

\section{Discussion and Conclusion}

Traumatic superficial femoral artery injuries secondary to femoral shaft fractures were rarely reported in the literature. Vascular 


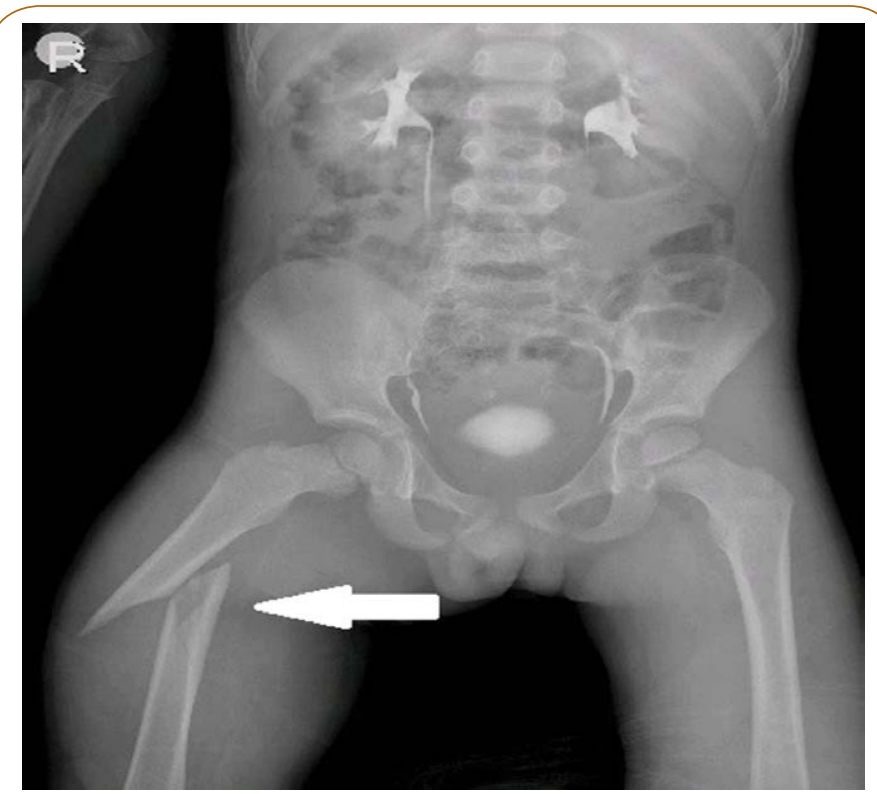

Figure 1 View of the right femoral shaft fracture on the direct graphy.

injury depends on the feature of the trauma. The medial aspect of the femur has a close relationship with the common femoral, superficial femoral and deep femoral arteries and veins. These arteries are more prone to vascular injury associated with femoral shaft fracture [3]. Atherosclerosis and arterial wall status are the major etiological risk factors. Major signs of the vascular injury are including pulselessness, enlarging or pulsatile haematoma, bruit, thrill, haemorrhage and acute ischemia. Doppler US, computed tomography angiography, magnetic resonance angiography can also be used for diagnosing, however, vascular injury can also be diagnosed with the physical examination. Digital subtraction angiography is still the gold standard diagnostic tool [4]. Bypass, proximal or distal thrombectomy can be performed for the treatment of vascular injuries. In conclusion, vascular injury may present even if in the presence of the distal pulses of the limb by collateral arterial flow. For this reason diagnoses of vascular injuries which are associated with femoral shaft fractures can be delayed [5]. Failure of prompt diagnosis of vascular injury and the severity of the soft-tissue damage are the potential reasons for the amputation. Groin infections and the lymphorrhea are the main disadvantages of this procedure; however, it is essential for the limb salvage.

The aim of the successful management is to save the limb by restore the circulation and repair of the bone. We emphasize the significance of suspicion of vascular injuries associated with femoral shaft fractures in pediatric population. 


\section{References}

1 Barquet A, Gelink A, Giannoudis PV (2015) Proximal femoral fractures and vascular injuries in adults: Incidence, aetiology and outcomes. Int J Care Injured 46: 2297-2313.

2 Al-Salmanl MMS, Al-Khawashki H, Sindigki A, Rabee H, Al-Saif A, et al. (1997) Vascular injuries associated with limb fractures. Injury 28: 103-107.
3 Kluger Y, Gonze MD, Paul DB (1994) Blunt vascular injury associated with closed mid-shaft femur fracture: A plea for concern. J Trauma 36: 222-225.

4 Russell GV, Kregor PJ, Jarrett CA, Zlowodzki M (2002) Complicated femoral shaft fractures. Orthop Clin North Am 33: 127-142.

5 Price KR, Hunter JB (2011) Lower limb injuries in children. Surgery 29: 162-166. 